\title{
Equivalent/generic drugs use. To whom? When? How?
}

\author{
Andrea Fontanella \\ OU Medicina Interna, AO Buon Consiglio, Fatebenefratelli, Napoli, Italy
}

In this issue of the journal, we publish three works by Nardi et al. ${ }^{1-3}$ on the use of generic/equivalent drugs in medical patients. The first part, including the definition of drug equivalent, presents the complex glossary used in this field, which is not adequately known among healthcare workers, and describes the regulatory framework concerning the use of drugs and the methods to determine the different equivalences between branded and generic drugs. The second part and the third part highlight other aspects, such as doubts, confusion, perplexity, lack of knowledge and biases, which in Italy limit the use of generics in the clinical practice.

Regulatory authorities argued that differences in systemic exposure to a drug up to $\pm 20 \%$ are not clinically significant. This tolerance of $\pm 20 \%$ is not related to the content of the active ingredient in the pharmaceutical preparation, but to its concentration in the blood. This range is related to the inter-and intra-individual biological variability. In general, it is reasonable to believe that the controls associated with the issuance of the autorizzazione all'immissione in commercio or marketing authorization for generics provide sufficient guarantees to ensure their interchangeability with the corresponding branded drug. However, in this field, there are still some problems that cannot be hidden, but are often underestimated or neglected. The bioequivalence tests, as they are made today, can provide for an assessment of the average bioequivalence of the population, but not individual bioequivalence. Allen Roses, vice president of the GlaxoSmithKline generic line, declared in 2003 that for most of the drugs that we pro-

Correspondence: Andrea Fontanella, Medicina Interna, Ospedale Buon Consiglio, Fatebenefratelli, via A. Manzoni 220, 80123 Napoli, Italy.

E-mail: andreafontanella52@gmail.com

Received for publication: 9 January 2014

Accepted for publication: 9 January 2014.

This work is licensed under a Creative Commons Attribution NonCommercial 3.0 License (CC BY-NC 3.0).

CCopyright A. Fontanella, 2014

Licensee PAGEPress, Italy

Italian Journal of Medicine 2014; 8:77-79

doi:10.4081/itjm.2014.472 duce - more than $90 \%$ - it works only in a proportion of between $30 \%$ and $50 \%$ of the people. ${ }^{4}$ According to this consideration, the doctor and the patient using an equivalent drug can expect an average equivalent therapeutic result in the overall population of users, but no information can be provided as to the likelihood that the patient's individual response to the two formulations of the reference drug and the generic one is the same. In other words, the authors argued that the estimated average bioequivalence does not measure the equivalence of the response to the two formulations (generic and brand) in individual subjects. It's true, on the other hand, that the effectiveness of a brand is evaluated on the population and not on the individual. The bioequivalence of the population, however, does not provide any information about the probability that the individual patient response can be equivalent in the two formulations. In order to capture this information, we need to assess the individual bioequivalence, i.e. the intra-subject bioequivalence, thus assessing the percentage of individuals who respond in an equivalent manner to the generic product and to its originator. Therefore the individual bioavailability emerges as the key criterion to apply the rule of substitutability between different formulations during treatment, without affecting the safety and the therapeutic profile obtained with the first formulation. The assessment of such individual bioavailability is extremely difficult and actually is not make in our country. In accordance with the rules on the bioequivalence studies, designed to minimize the extent of inter-individual variability and reduce the possibility of errors, the drug is tested on healthy volunteers aged between 18 and 55, in order to avoid interactions with the disorder. They cannot smoke or take drugs, must eat standardized meals to exclude that co-administration of food may interfere with the absorption of the generic drug under study. However these conditions are exactly the opposite compared with what occurs to real patients, who are complex patients with multiple disorders, older than 55 years, take various drugs, have a large variability in terms of food intake, a functional impairment of the main excretory organs, etc.

Another problem is that bioequivalence tests are carried out between the single generic product and the branded product. This approach does not ensure that two or more generic formulations of the same brand are equally bioequivalent to each other. For example, as- 
suming that a generic has a bioavailability or are under the concentration equal to $+15 \%$ and another generic has a bioavailability equal to $-15 \%$; they are certainly both bioequivalent with respect to the standard that they imitate, but not between themselves. Essentially the transitive property cannot be applied to the concept of bioequivalence: it is not possible to conclude indirectly that two products, each bioequivalent with the same standard of reference, are bioequivalent to each other. Unfortunately, a direct comparison is not possible as generics are compared only with the originator medicine, and so the interchangeability between equivalents is only supposed. This issue has neither been addressed in Italy nor in Europe yet, where we have to system to compare bioequivalence. On the contrary, the United States can rely on the Orange Book, which is periodically updated and reports all the bioequivalence studies that indicate for each generic the corresponding drugs that can be replaced. The problem is not insignificant, considering that the decision-maker in the choice of the equivalent drug is often the pharmacist who, when the prescribing physician does not explicitly indicate that the prescribed drug is not replaceable (notion of nonsubstitutability), may change the drugs, even several times in a row, without having a full understanding of the clinical problems of the patient and no certainty as to the bioavailability of the equivalent drug.

In prescribing and replacing the generic medicines we have to guarantee all potential economic benefits to both the healthcare system and the patients, without adopting any opportunistic approach related to higher discounts on some products than others, also branded. The cheapest drug does not always offer necessarily the best quality assurance. We wish to emphasize the need for better quality control on good manufacturing processes on generic medicines. This is all the more important in our current globalized world, where drug production is being shifted towards cheap labor countries, such as India and China, where however controls are uncertain and difficult. ${ }^{5}$

When prescribing an equivalent drug, one needs to consider the characteristics of the active ingredient, of the patient and her/his disease. For example, for drugs intended for continuous use and with a narrow therapeutic index, the conventional bioequivalence interval may be inadequate to provide sufficient confidence that the two bioequivalent products are also therapeutically equivalent, with the risk of incurring in a lack of efficacy or in excessive side effects, like in the case of antiepileptic drugs, anticoagulants, antiplatelet agents, antiarrhythmics, some antibiotics, etc. Therefore, although the concept of substitutability between the reference and the generic drug is generally valid, it is evident that for physicians it is important to know, for each alternative, the range variance of benchmarks, in order to choose the drug is the clos- est to the reference product. According to the principle of evidence-based medicine, there is no class effect, there is no transitive property in the indications between molecules of the same family. In order to validate an indication for a specific treatment, each molecule has to undergo phase three clinical trials for that specific indication. As a result, for example, all angiotensin-receptor blockers and angiotensin-converting enzyme inhibitors are indicated for the treatment of hypertension, but only some of them have indications for heart failure, post-myocardial infarction, prevention of kidney damage in diabetic patients, prevention of cardiovascular risks. The same applies to many other drugs, such as antiplatelet agents, antidepressants, and so on. Therefore, besides the complex, but precise and codified (and better known) regulation for branded medicines, we have another regulation concerning generic drugs, which is not always well known and prescribes that the technical data sheets must inform about the absolute non-uniformity of the therapeutic indications.

Most generics have not the same indications marked on the data sheet of the branded product and indications are often different from generic to generic. For all these reasons, I am not so confident that, according to the statement of the Italian Drug Agency (Agenzia Italiana del Farmaco - AIFA) the prescription of a medicinal product in place of another does not constitute an off-label prescription, because the substitution of an originator with its generic is based on documentation of bioequivalence and not on randomized control trials that led to the recognition of the originator drug.

I wish to recall that the regulatory requirements for prescribing pharmaceuticals foresee that the doctor in prescribing a medicinal product complies with the therapeutic indications, and the ways and methods of administration provided by marketing authorization (D.lgs. 23/1998, converted into Law 94/1998, art. 3 observance of the authorized the therapeutic indications). ${ }^{6}$

For the purpose of an on label prescription for all drugs, what matters are the indications on the datasheet. If the indications defined by the European Medicines Agency can be changed, why does AIFA not harmonize at the same time these indications in Italy too? Why does any datasheet of generics not report the same technical details and indications as those of the originator? In the case of legal controversies which is the relevance of AIFA opinions?

A few remarks on the issue of excipients: the existing law, based on D.L. 323 of 1996 states that generics must have the same qualitative and quantitative composition in active substances and the same pharmaceutical form as the same therapeutic indication. ${ }^{7}$ The law does not provide for the composition of the excipients. The problem is not insignificant, particu- 
larly with regard to pharmaceutical forms such as granules, oral solutions, tablets, capsules, dermatological preparations. This does not only entail problems in terms of bioavailability of the active principle, but also of general or specific allergies or intolerances to various types of substances. The increased incidence of diseases that require dietary restrictions or the elimination of certain substances, is leading us to pay greater and greater attention to this problem.

The manuscript concludes that health authorities and politicians tend to encourage the use of generic drugs, primarily for economic reasons, and that the substitution of branded products for generic drugs is still very controversial and not free from bias and uncertainties on the part of both healthcare professionals and patients. Generic drugs provide an excellent opportunity for increasing the economic sustainability of the healthcare system. Indeed actual prices of branded drugs are remarkably tiered to withstand the impact of competitive generics. Anyhow, despite the local healthcare units reimburse the cost of generic drugs, many users choose to purchase the brand-name drug. Therefore the burden of the extra cost is borne by citizen and not by the state. This policy is controversial and may not be accepted from a demagogic the point of view, but it is a reality. Current national regulations impose strict and well-defined rules on bioequivalence. Unfortunately this is not enough to ensure that these rules are complied with. Who exercises this control, how is it done, when implementing it, about who runs it? The paradox that in southern Italy, which is the poorest part of the country, patients use fewer equivalent drugs than in the richest regions of the North probably shows less confidence in the public institutions and in the controllers, unlike what happens (perhaps rightly) in northern Italy.

The choice to promote the use of generics, given this significant complexity, cannot only be dictated by a single goal, such as cost reduction, but also by the credibility of the quality control and substitutability of the products offered to replace the same molecule. Doctors, together with pharmacists and research pharmacologist have to find solutions for unsolved problems and unanswered questions, through specific studies, communication tools and shared guidelines. It is strictly necessary to focus on the quality of generics, as happens in Germany with Ratiopharm, or by adopting a tool such as the North American Orange Book. To avoid unfair competition we must prevent generic companies from offering disproportionate discounts and have them reinvest in the quality control of their products. Most generic drugs currently available in Italy are very handy. If generic drugs do not affect the innovation cycle and the interests of the individual patients, it can certainly offer a tangible reduction in expenditure and the possibility of allocating savings to other issues. For this reason, it is necessary to comply painstakingly with good rules and monitor compliance with them. It is also equally important to ensure the appropriate use of drugs, i.e. the right drug to the right patient at the right time. Each adequately informed patient has the right to receive the most suitable treatment depending on her/his expectations and her/his biological and clinical specificity.

\section{References}

1. Nardi R, Masina M, Cioni G, et al. Generic - equivalent drugs use in internal and general medicine patients: distrust, confusion, lack of certainties or of knowledge? Part 1. Pharmacological issues. Ital J Med 2014;2:80-7.

2. Nardi R, Masina M, Cioni G, et al. Generic - equivalent drugs use in internal and general medicine patients: distrust, confusion, lack of certainties or of knowledge? Part 2. Misconceptions, doubts and critical aspects when using generic drugs in the real world. Ital J Med 2014;2: 88-98.

3. Nardi R, Masina M, Cioni G, et al. Generic - equivalent drugs use in internal and general medicine patients: distrust, confusion, lack of certainties or of knowledge? Part 3. Clinical issues. Ital J Med 2014;2:99-109.

4. Connor S. Glaxo chief: our drugs do not work on most patients. The Independent; 8 December 2003. Available from: http://www.independent.co.uk/news/science/glaxochief-our-drugs-do-not-work-on-most-patients-575942. html

5. European Medicines Agency. European Medicines Agency recommends precautionary recall of batches of clopidogrel-containing medicines from Acino Pharma $\mathrm{GmbH}$ - Recall due to good manufacturing practice (GMP) failure at active substance manufacturer site. EMA/179606/2010; 25 March 2010. Available from: http://www.ema.europa.eu/docs/en_GB/document_library/Press_release/2010/03/WC500076546.pdf

6. Ministero della Sanità Italiana. Testo del decreto-legge 17 febbraio 1998, n. 23 (in Gazzetta Ufficiale - serie generale - n. 39 del 17 febbraio 1998), coordinato con la legge di conversione 8 aprile 1998, n. 94, recante: "Disposizioni urgenti in materia di sperimentazioni cliniche in campo oncologico e altre misure in materia sanitaria”. In: G.U. Ser. Gen. n. 86, 14/4/1998, pp 29-31. Available from: http://www.agenziafarmaco.gov.it/sites/default/files/di bella.pdf

7. Presidente della Repubblica Italiana. Decreto legge 20 giugno 1996, n. 323 disposizioni urgenti per il risanamento della finanza pubblica. In: G.U. Ser. Gen. n. 143, 20/6/1996. Available from: http://www.gazzettaufficiale. it/eli/id/1996/06/20/096G0348/sg;jsessionid=20yoJZim5i rcacOi+rZF1g_.ntc-as2-guri2a 